\title{
Vaccine Refusal Is Not Free Riding
}

\author{
ETHAN BRADLEY \\ Oakland University \\ MARK NAVIN \\ Oakland University
}

\begin{abstract}
Vaccine refusal is not a free rider problem. The claim that vaccine refusers are free riders is inconsistent with the beliefs and motivations of most vaccine refusers. This claim also inaccurately depicts the relationship between an individual's immunization choice, their ability to enjoy the benefits of community protection, and the costs and benefits that individuals experience from immunization and community protection. Modeling vaccine refusers as free riders also likely distorts the ethical analysis of vaccine refusal and may lead to unsuccessful policy interventions.
\end{abstract}

Keywords: fairness, free riding, game theory, public health, vaccination

JEL Classification: C72, I18

\section{INTRODUCTION}

The Covid-19 pandemic has highlighted problems of non-compliance with public health guidelines. Some people refuse to wear masks in public, to engage in social distancing, or even to avoid large gatherings (Breslow 2020; Glanz et al. 2020; van Rooij et al. 2020). Of particular interest to us is that some have indicated an unwillingness to receive Covid-19 vaccines (Freeman et al., forthcoming; Lazarus et al. 2021). Refusal of Covid-19 vaccines would therefore seem to be another instance of the general phenomenon of vaccine refusal, which has recently contributed to outbreaks of previously well-controlled infections (Jacobson, St. Sauver, and Finney Rutten 2015; Phadke et al. 2016).

Researchers have sometimes characterized vaccine refusal as a free rider problem (Bauch, Bhattacharyya, and Ball 2010; Betsch, Böhm, and Korn 2013; Betsch et al. 2017; Buttenheim and Asch 2013; van den Hoven 
2012; May and Silverman 2005; Schröder-Bäck et al. 2009; Siegal, Siegal, and Bonnie 2009). That is, they have claimed that vaccine refusers promote their individual interest by benefitting from the community's protection from infection without also making a contribution to the community's protection by becoming vaccinated themselves. ${ }^{1}$ Notably, this characterization of vaccine refusers applies only in cases in which the community already has protection against infection-that is, when a sufficiently large percentage of the population, having been vaccinated, is immune to the disease so that outbreaks are unlikely. Most countries possess community protection for many vaccine-preventable diseases, including, for example, polio (Global Polio Eradication Initiative n.d.). Though none yet have community protection against Covid-19, we may hope that ongoing vaccine distribution efforts will soon generate it.

The diagnosis of vaccine refusal as a kind of free riding is supposed to inform our understanding of the origins of vaccine refusal (vaccine refusers rationally pursue their own interests), the ethical analysis of vaccine refusal (vaccine refusers make unfair use of community protection), and potential policy responses (vaccine refusers will vaccinate if one makes slight changes to their incentives).

But, as we shall argue, vaccine refusal is not a kind of free riding. First, a free rider model misrepresents the subjective motivations of most vaccine refusers. Vaccine refusers often doubt that vaccines provide benefits to individuals and communities, and often radically overstate the risks associated with vaccination. That is, vaccine refusers do not think they are using a valuable public good (community protection) or that they are refusing to make a reasonable contribution to that good since they think the expected costs of vaccination are very high. Second, a free rider model misstates the objective relationship between individual vaccination choices and one's ability to benefit from community protection. A free rider benefits from a public good that they could also be contributing to, but it is not possible to both contribute to community protection and to benefit from it. This is because the means by which a person contributes to community protection (individual immunity, usually generated through vaccination) makes it impossible for that same person to benefit

\footnotetext{
${ }^{1}$ We follow contemporary usage in referring to 'community protection', rather than 'herd immunity', because the community is protected rather than immune and because we are speaking of a moral community of human persons rather than a passive animal herd (Anderson et al. 2018).
} 
from community protection. ${ }^{2}$ Furthermore, the act of 'contributing' to community protection (by becoming vaccinated) is, by itself, individually rational, even when community immunization rates are high, because there is rarely an objective anticipated cost of vaccination for individuals. Free riding is individually rational, by definition, but refusing vaccines rarely is.

\section{VACCINE REFUSAL AS FREE RIDING}

An individual is a free rider if they enjoy the benefits of a public good (that is, a good that is non-rivalrous and non-excludable) without also contributing to it, even though they could also contribute to the good, and even though the personal benefits they acquire from the public good would outweigh their personal costs of contribution (Olson 1965). Free riding creates a collective action problem: it is individually rational to free ride, but if enough people free ride, then no one can, since insufficient contribution undermines or destroys the good that they were attempting to enjoy. That is a collectively irrational outcome. In this way, free riding problems may take the shape of a Prisoner's Dilemma, wherein a social good can be created by cooperation, but each individual benefits from defecting (Hardin 1971).

Furthermore, some have argued that free riding is ethically wrong when the public good is sufficiently valuable and the costs of contribution are sufficiently low. On this kind of view, free riding is not only collectively irrational, but also individually unethical, because it is unfair to benefit from the contributions of others without making a reasonable contribution oneself (Cullity 1995).

If vaccine refusal were a kind of free riding, then (1) mass vaccination must create a public good, (2) it must be possible for individuals to contribute to that good and also to benefit from it, though (3) it must be better for individuals not to contribute, but just to benefit from it. Furthermore, vaccine refusers would have an intention to free ride just in case they intended to enjoy the benefits of the public good that mass vaccination creates, but they did not intend to pay the (small) costs associated with contributing to that good.

\footnotetext{
An individual can (1) intend to contribute to community protection (by getting vaccinated) without actually contributing to community protection (if the vaccine does not give them immunity), and (2) continue to benefit from community protection. But such a person does not both contribute to and benefit from community protection.
} 
Mass vaccination creates a public good. 'Community protection' (sometimes called 'herd immunity') exists when a community has a sufficiently high rate of individual immunity, such that outbreaks are highly unlikely and such that it is highly improbable that individuals who are not immune to infection will become infected (Anderson et al. 2018). The logic of free riding on community protection may seem straightforward: vaccination has expected costs for individuals, including time, money, and risks of side effects and adverse events. Community protection has many expected benefits for individuals, including decreased morbidity and mortality. The benefits an individual enjoys from community protection outweigh the individual costs associated with vaccination. However, someone who free rides on community protection enjoys the benefits of community protection without paying any costs associated with contributing to it.

Scholars writing about empirical issues in vaccine refusal have sometimes modeled vaccine refusal as a form of free riding (Bauch, Bhattacharyya, and Ball 2010; Betsch, Böhm, and Korn 2013; Betsch et al. 2017; Buttenheim and Asch 2013; van den Hoven 2012; Ibuka et al. 2014; May and Silverman 2005; Siegal, Siegal, and Bonnie 2009). The details of their models differ. For example, Bauch and colleagues (2010) show that free riding practices can develop quickly after the introduction of a vaccine that is universally and freely available but not mandatory. For another example, Ibuka and colleagues (2014) found that individuals were less likely to vaccinate in a simulation when the rate of vaccination was high in the previous round of the simulation. But the common thread that ties together 'free riding' models of vaccine refusal is that vaccine refusers pursue their rational self-interest in avoiding the costs associated with vaccination while benefiting from the vaccination behaviors of others.

People who have written about the ethical issues involved in vaccine refusal have also sometimes invoked the idea that refusers are free riders who make selfish and unfair use of others' contributions to community protection (Clarke, Giubilini, and Walker 2017; Dawson 2007; Giubilini, Douglas, and Savulescu 2017; Giubilini 2019; Hendrix et al. 2016; van den Hoven 2012; Navin 2016; Salmon and Siegel 2001). Some have argued that one goal of immunization policy is to fairly distribute the burden of achieving community protection while avoiding unfair free riding. For example, they have proposed that nonmedical exemptions to vaccine mandates only be granted to people who make an alternative contribution to public health-that is, contributions similar to those of some conscripted 
pacifists who are asked to provide alternative (for example, non-combatant) services to a war effort (Clarke, Giubilini, and Walker 2017; Giubilini, Douglas, and Savulescu 2017).

\section{WHY NOT FREE RIDING?}

Vaccine refusal is not a case of free riding. Vaccine refusers do not have the subjective beliefs and attitudes of free riders, and the objective costs and benefits associated with vaccine refusal are not consistent with free riding.

\section{III.I. Beliefs and Attitudes}

Vaccine refusers rarely possess the beliefs and attitudes towards vaccination and community protection that are consistent with them being subjective free riders. Someone intends to free ride when they recognize the benefits of a public good that they are enjoying and when they acknowledge that they are benefitting from that good without making a reasonable contribution to it. For example, in Prisoner's Dilemma models, a defector (who wishes to be a free rider) seeks to take advantage of the benefits of other people's cooperative behavior without themselves engaging in cooperative behavior. Vaccine refusers do not fit this profile. First, vaccine refusers often believe that vaccines are ineffective, that vaccines do not cultivate individual immunity to disease, and that community protection does not exist (Harmsen et al. 2013; Sobo 2016). Others are wholly unfamiliar with the concept of community protection (QuadriSheriff et al. 2012; Sobo 2016). Such vaccine refusers cannot be motivated by a desire to take advantage of others' cooperative behavior because they do not think other people's vaccination choices create a public good. There are some exceptions. For example, Dr. Bob Sears recommends that parents who refuse vaccines for their children do not "share their fears with their neighbors, because if too many people avoid the MMR [measles, mumps, and rubella vaccine], we'll likely see the disease increase significantly" (Sears 2007, 96-97). The clear implication is that parents of children in Sears' practice should want other parents to continue to vaccinate so that their own children will be safe from disease. But it is rare to see vaccine refusers acknowledge that they are benefitting from other people's decisions to vaccinate. A more common view is that vaccines are ineffective, that they are collectively harmful, and that everyone would be better off if no one were vaccinated (Dubé et al. 2013; Harmsen et al. 2013; Sobo 2016). 
Second, part of the idea of free riding is that it better promotes an individual's interests than contribution does, but that contributing to (and enjoying) a public good is better for the individual than non-contribution and non-enjoyment of the public good would be. This is because an individual's costs of contribution are outweighed by the benefits they receive from the public good. So, even though free riding on a public good is individually rational, contribution is also beneficial (though less so than free riding) compared to not enjoying the public good at all. In the case of modeling vaccine refusal as free riding, a free rider would better promote their own interests than would someone who were vaccinated, but a vaccinated person enjoying community protection would better promote their own interests than would someone who did not benefit from community protection at all. On such a view, an individual's expected costs of vaccination are more than compensated for by the benefits they receive from community protection.

But many vaccine refusers do not believe that the expected costs of vaccination are low. They reject the scientific consensus that vaccines usually have only mild side effects and that the risks of serious adverse events (for example, death or life-threatening illness) are very low (Smith 2015). Vaccine refusers commonly believe that vaccines often cause serious disorders (including autism), damage one's immune system, degrade one's genetic code, or place one's body under unacceptable forms of government surveillance or control (Dubé et al. 2013). Someone who intends to free ride aims to avoid paying a reasonable price for a public good. But many vaccine refusers think that vaccination is dangerous and that it would be unreasonable to ask them to vaccinate. Such people conceive of vaccination choices as a matter of avoiding or acquiescing to significant harms, rather than as a matter of whether or not to contribute to a public good from which they benefit.

\section{III.II. Costs and Benefits}

Vaccine refusal also usually fails to meet objective criteria for free riding. First, vaccine refusers are not free riders on community protection, because it is not possible to both contribute to and benefit from community protection. A free rider benefits from a public good to which they could also contribute. When free riding is immoral, free riders should also contribute to the public goods from which they benefit. The way to contribute to community protection is to possess individual immunity, either through vaccination or by recovering from a disease. But people who 
possess individual immunity do not (and indeed, cannot) rely on community protection to protect themselves from the diseases against which they have individual immunity. They rely on individual immunity. Accordingly, someone who contributes to community protection cannot also benefit from it. Consequently, someone who does not contribute to community protection-for example, by refusing vaccines-is not a free rider.

Second, free riding is individually rational, but vaccine refusal is not. Vaccination almost always promotes the interests of the vaccinated individual, even at very high levels of community protection. Since the costs of vaccination are generally negligible-for example, serious complications are exceedingly rare (Spencer, Trondsen Pawlowski, and Thomas 2017) - it is almost always in a person's interest to vaccinate, even when community protection makes their odds of infection very low. Consider the fact that community protection against some vaccine-preventable infections requires very high levels of population-level immunity, such that even universal vaccination with very effective vaccines may still leave someone vulnerable to exposure (and therefore make it rational to be vaccinated). For example, roughly $95 \%$ of the population needs to be individually immune to measles to eliminate outbreaks (Gay 2004; Moss and Strebel 2011), while only $95 \%$ of people fully vaccinated against measles have individual immunity (Demicheli et al. 2012), so that communities with less than $100 \%$ measles vaccine uptake remain at risk for measles outbreaks, and therefore individual vaccination against measles is almost always rational (Bester 2017). More importantly, it is very difficult to know one's risk of being exposed to a vaccine-preventable infection, even if reliable community-wide immunization rates are available. Outbreaks commonly appear in small geographically clustered groups of under-vaccinated persons (Omer et al. 2008; Phadke et al. 2016), and it can be all but impossible to identify and avoid such groups. In contrast, vaccination reliably generates individual immunity at negligible costs. Notably, attempts to model vaccine refusal as an instance of free riding sometimes presume that individuals can make reliable predictions about the proportion of immune individuals in the groups they interact with (for example, Betsch, Böhm, and Korn 2013). Yet, it seems highly unlikely that most vaccine refusers possess (or even could possess) such knowledge.

\section{IV. 'Free Riding' AND Policy InTERVENTIONS}

If policy responses to vaccine refusal are to be effective and ethically justified, then they should be based on an accurate understanding of vaccine 
refusal. The claim that vaccine refusers are free riders may lead to policy proposals that are ineffective or morally unjustified.

\section{IV.I. Incentives and Pro-Vaccine Interventions}

There are a set of standard interventions for solving free riding problems (Buttenheim and Asch 2013). These include offering economic incentives for cooperation (or disincentives for defection), restricting defectors' access to other goods, invoking or creating social norms to pressure individuals to cooperate, or using state coercion. These enforcement mechanisms shift the relevant payoffs in ways that incentivise potential free riders to cooperate, instead of defect, by making it rational for would-be free riders to contribute (Buttenheim and Asch 2013).

If vaccine refusal were a free rider problem, then one way to encourage vaccination would be to slightly shift the perceived payoffs of potential vaccine refusers. Governments should be able to avoid vaccine refusal with small (or even trivial) amounts of incentives or disincentives. Recall that actual free riders acknowledge the value of the public good they enjoy but refuse to pay the small cost of contributing to that good when they can get away with not incurring the cost. A government could therefore overcome 'free rider vaccine refusal' if it increased the costs of vaccine refusal so that those costs were somewhat higher than the costs of vaccination. This means that placing small additional burdens on vaccine refusers should lead to overcoming vaccine refusal that results from subjective free riding.

The evidence is not consistent with the hypothesis that the behavior of most vaccine refusers can be changed by making small modifications to their incentives. For example, many countries have recently adopted or revised childhood vaccine mandates as a way of providing additional incentives for vaccination or of disincentivizing vaccine refusal. For example, Australia's federal government withholds state payments to parents who do not vaccinate their children ('No Jab, No Pay'), while their state governments prevent unvaccinated children from being enrolled in childcare ('No Jab, No Play') (Attwell et al. 2018). All US states require children to be vaccinated to enroll in school, and many states have recently made those enrollment mandates more difficult to escape, for example, by eliminating nonmedical exemptions or imposing burdensome administrative procedures (National Conference of State Legislatures 2021). Other political communities, like Italy, have decided to fine parents who do not vaccinate their children (Vaz et al. 2020). 
These kinds of policy changes sometimes somewhat increase vaccination rates or decrease nonmedical exemption rates (Omer et al. 2012; Navin, Largent, and McCright 2020). That is not evidence that the parents who change their minds in the face of such changed payoffs are free riders-we have already presented evidence to motivate skepticism about that conclusion-but the number of parents who do change their behaviors clearly identifies a ceiling for the maximum number of possible free riders among vaccine refusers. Even if we supposed that every parent who changed their vaccination behavior in the face of changes in their incentive structure were a free rider, then the evidence suggests that perhaps around a third of parents could possibly be free riders-that is about the maximum number of vaccine refusers who seem willing to change their behavior in the face of substantial changes to their incentives. Notably, even quite serious penalties seem insufficient to change the behavior of many vaccine refusers. For example, Delamater and colleagues (2019) found that eliminating nonmedical exemptions in California caused few parents to vaccinate their children. In fact, the positive results of that policy change were almost entirely a consequence of better administrative oversight and record-keeping, or were offset by corresponding increases in medical exemptions or the enrollment of children not in compliance with immunization requirements. Many thousands of California parents allowed their children to be denied access to public or private schools, or to be prevented from participating in group homeschooling activities rather than be vaccinated. This is not the profile of a free rider.

Pro-vaccination policies that are likely to overcome free riding behavior, especially subjective free riding behavior, are unlikely to be as effective against someone who refuses vaccines because they believe vaccines are ineffective or dangerous. If someone does not think that community protection is valuable, or if they think vaccination is a grave risk to their health, then small changes to their incentive structure are unlikely to change their minds. Relying on a free rider model of the attitudes and motivations of vaccine refusers may therefore encourage unjustified optimism that vaccine refusal can be overcome with minimally invasive interventions.

\section{IV.II. Ethical Justifications for Pro-Vaccine Interventions}

The mere fact that community protection is a public good does not, by itself, justify government coercion (Bernstein and Randall 2020), but the substantial individual and collective benefits of community protection 
strongly favor government efforts to promote vaccination (Brennan 2018; Flanigan 2014; Giubilini and Savulescu 2019; Giubilini 2019, 2020; Navin 2016; Pierik 2018). If vaccine refusal were objectively a form of free riding, then one weighty ethical reason for societies to promote vaccination would be to promote fairness in the distributions of benefits and burdens associated with mass immunization. The people who benefit from community protection should be incentivized or even compelled to contribute to community protection, rather than be allowed to unfairly free ride on the socially productive contributions of others, as long as they can do so at a reasonable cost to themselves.

But vaccine refusers are not objectively free riders, even though some of them may think they are free riding, as in the case of Dr. Bob Sears' patients we discussed above. The goal of pro-vaccination policies, therefore, should not be to punish people for free riding, that is, to make sure that they 'pay' for the benefits that they enjoy from community protection. As we argued above, it is not possible both to contribute to and to benefit from community protection. Inasmuch as fairness plays a role in ethical arguments for pro-vaccination policies, it should be to make sure that there is a fair distribution of labor among the members of society who have a duty to protect others from harm (Giubilini, Douglas, and Savulescu 2018). That is, fairness in vaccination behavior is about doing one's part to help others. It is about being a contributor to community protection, rather than a beneficiary of it, whenever one is reasonably able to do so. But fairness with respect to vaccination is not about ensuring that one is paying for the public goods they are benefiting from: this is not possible.

Aside from the fact that vaccine refusal is not objectively a free rider problem, it also matters for an ethical analysis that vaccine refusers are rarely subjectively free riders. Vaccine refusers usually do not think they are acting unfairly. They do not believe they are taking advantage of a public good that they could also be contributing to. Instead, they think that (almost) everyone would be better off if they refused vaccines. So, inasmuch as vaccination policies aim to punish vaccine refusers for their unethical behavior, it would be incorrect to focus on refusers' supposed intentions to free ride. Relatedly, inasmuch as vaccine advocates express anger at vaccine refusers for (what they believe to be) unfair free riding (Bernstein 2021), those moral emotions are not entirely apt. Vaccine refusers seem to be motivated by ordinary ideas about avoiding serious risks to their health based on false beliefs about the costs and benefits of 
vaccination and about the risks of vaccine-preventable infections. We acknowledge that vaccine refusers may sometimes be morally responsible for these false beliefs and that they may be acting unfairly, but their moral failure is not the straightforward unfairness of free riding.

\section{CONCLUSION}

Many people have endorsed pro-vaccination interventions or made ethical arguments about vaccine refusal that presume vaccine refusers are free riders. We have argued that vaccine refusal is not an instance of free riding and that few vaccine refusers believe themselves to be free riding. Accordingly, effective policy interventions and apt ethical judgments about vaccine refusal will need to rely on other explanations for why people sometimes refuse vaccines.

If those who study vaccine refusal want to introduce game-theoretic models to explain this phenomenon, then perhaps they should turn away from free rider models and consider embracing information problem models. The core dynamics of information problem game-theoretic models is that participants in the game are not fully aware of the 'rules' of the game, for example, who else is playing, what each participant's interests are, and what actions have been taken previously (Jones 1977). Participants must therefore make educated guesses about the best course of action, rather than calculate a deterministic result. In light of the prominent role that false beliefs play in the decisions of vaccine refusers, such models may present more accurate depictions of the objective and subjective attributes of vaccine refusal than free rider models do.

\section{REFERENCES}

Anderson, Evan J., Michael A. Daugherty, Larry K. Pickering, Walter A. Orenstein, and Ram Yogev. 2018. "Protecting the Community through Child Vaccination." Clinical Infectious Diseases 67 (3): 464-471.

Attwell, Katie, Mark C. Navin, Pier Luigi Lopalco, Christine Jestin, Sabine Reiter, and Saad B. Omer. 2018. "Recent Vaccine Mandates in the United States, Europe and Australia: A Comparative Study." Vaccine 36 (48): 7377-7384.

Bauch, Chris T., Samit Bhattacharyya, and Robert F. Ball. 2010. "Rapid Emergence of FreeRiding Behavior in New Pediatric Immunization Programs.” PLoS ONE 5 (9): e12594.

Bernstein, Justin. 2021. “Anti-Vaxxers, Anti-Anti-Vaxxers, Fairness, and Anger.” Kennedy Institute of Ethics Journal 31 (1): 17-52.

Bernstein, Justin, and Pierce Randall. 2020. "Against the Public Goods Conception of Public Health.” Public Health Ethics 13 (3): 225-233.

Bester, Johan Christiaan. 2017. "Measles Vaccination Is Best for Children: The Argument for Relying on Herd Immunity Fails.” Journal of Bioethical Inquiry 14 (3): 375-384. 
Betsch, Cornelia, Robert Böhm, and Lars Korn. 2013. "Inviting Free-Riders or Appealing to Prosocial Behavior? Game-Theoretical Reflections on Communicating Herd Immunity in Vaccine Advocacy." Health Psychology 32 (9): 978-985.

Betsch, Cornelia, Robert Böhm, Lars Korn, and Cindy Holtmann. 2017. "On the Benefits of Explaining Herd Immunity in Vaccine Advocacy." Nature Human Behaviour 1 (3): 0056.

Brennan, Jason. 2018. “A Libertarian Case for Mandatory Vaccination.” Journal of Medical Ethics 44 (1): 37-43.

Breslow, Jason. 2020. "Fauci: Mixed Messaging on Masks Set U.S. Public Health Response Back.” National Public Radio, July 1, 2020. https://www.npr.org/sections/healthshots/2020/07/01/886299190/it-does-not-have-to-be-100-000-cases-a-day-fauciurges-u-s-to-follow-guidelines.

Buttenheim, Alison M., and David A. Asch. 2013. "Making Vaccine Refusal Less of a Free Ride." Human Vaccines \& Immunotherapeutics 9 (12): 2674-2675.

Clarke, Steve, Alberto Giubilini, and Mary Jean Walker. 2017. "Conscientious Objection to Vaccination." Bioethics 31 (3): 155-161.

Cullity, Garrett. 1995. “Moral Free Riding.” Philosophy \& Public Affairs 24 (1): 3-34.

Dawson, Angus. 2007. "Herd Protection as a Public Good: Vaccination and Our Obligations to Others." In Ethics, Prevention, and Public Health, edited by Angus Dawson and Marcel F. Verweij, 160-178. Oxford: Clarendon Press.

Delamater, Paul L., S. Cassandra Pingali, Alison M. Buttenheim, Daniel A. Salmon, Nicola P. Klein, and Saad B. Omer. 2019. "Elimination of Nonmedical Immunization Exemptions in California and School-Entry Vaccine Status.” Pediatrics 143 (6): e20183301.

Demicheli, Vittorio, Alessandro Rivetti, Maria Grazia Debalini, and Carlo Di Pietrantonj. 2012. "Vaccines for Measles, Mumps, and Rubella in Children." Cochrane Database of Systematic Reviews 2: CD004407.

Dubé, Eve, Caroline Laberge, Maryse Guay, Paul Bramadat, Réal Roy, and Julie A. Bettinger. 2013. "Vaccine Hesitancy: An Overview." Human Vaccines \& Immunotherapeutics 9 (8): 1763-1773.

Flanigan, Jessica. 2014. “A Defense of Compulsory Vaccination.” HEC Forum 26 (2): 525.

Freeman, Daniel, Bao S. Loe, Andrew Chadwick, Cristian Vaccari, Felicity Waite, Laina Rosebrock, Lucy Jenner et al. Forthcoming. "COVID-19 Vaccine Hesitancy in the UK: The Oxford Coronavirus Explanations, Attitudes, and Narratives Survey (Oceans) II." Psychological Medicine.

Gay, Nigel J. 2004. "The Theory of Measles Elimination: Implications for the Design of Elimination Strategies.” The Journal of Infectious Diseases 189 (Supplement_1): S27S35.

Giubilini, Alberto. 2019. The Ethics of Vaccination. Cham: Palgrave Macmillan.

Giubilini, Alberto. 2020. "An Argument for Compulsory Vaccination: The Taxation Analogy.” Journal of Applied Philosophy 37 (3): 446-466.

Giubilini, Alberto, and Julian Savulescu. 2019. "Vaccination, Risks, and Freedom: The Seat Belt Analogy." Public Health Ethics 12 (3): 237-249.

Giubilini, Alberto, Thomas Douglas, and Julian Savulescu. 2017. "Liberty, Fairness and the 'Contribution Model' for Non-Medical Vaccine Exemption Policies: A Reply to Navin and Largent." Public Health Ethics 10 (3): 235-240. 
Giubilini, Alberto, Thomas Douglas, and Julian Savulescu. 2018. "The Moral Obligation to Be Vaccinated: Utilitarianism, Contractualism, and Collective Easy Rescue." Medicine, Health Care and Philosophy 21 (4): 547-560.

Glanz, James, Benedict Carey, Josh Holder, Derek Watkins, Jennifer Valentino-DeVries, Rick Rojas, and Lauren Leatherby. 2020. “Where America Didn't Stay Home Even as the Virus Spread.” The New York Times, April 2, 2020. https://www.nytimes.com/interactive/2020/04/02/us/coronavirus-social-distancing.html.

Global Polio Eradication Initiative. n.d. "Polio-Free Countries." Global Polio Eradication Initiative. Accessed January 23, 2021. https://polioeradication.org/where-we-work/ polio-free-countries/.

Hardin, Russell. 1971. “Collective Action as an Agreeable n-Prisoners' Dilemma.” Behavioral Science 16 (5): 472-481.

Harmsen, Irene A., Liesbeth Mollema, Robert A. C. Ruiter, Theo G. W. Paulussen, Hester E. de Melker, and Gerjo Kok. 2013. "Why Parents Refuse Childhood Vaccination: A Qualitative Study Using Online Focus Groups.” BMC Public Health 13 (1): 1183.

Hendrix, Kristin S., Lynne A. Sturm, Gregory D. Zimet, and Eric M. Meslin. 2016. "Ethics and Childhood Vaccination Policy in the United States." American Journal of Public Health 106 (2): 273-278.

Ibuka, Yoko, Meng Li, Jeffrey Vietri, Gretchen B. Chapman, and Alison P. Galvani. 2014. "Free-Riding Behavior in Vaccination Decisions: An Experimental Study." PLoS ONE 9 (1): e87164.

Jacobson, Robert M., Jennifer L. St. Sauver, and Lila J. Finney Rutten. 2015. "Vaccine Hesitancy." Concise Review for Clinicians 90 (11): 1562-1568.

Jones, Neil D. 1977. "Blindfold Games are Harder than Games with Perfect Information." DAIMI Report No. PB-80. Department of Computer Science, University of Aarhus, Denmark.

Lazarus, Jeffrey V., Scott C. Ratzan, Adam Palayew, Lawrence O. Gostin, Heidi J. Larson, Kenneth Rabin, Spencer Kimball, and Ayman El-Mohandes. 2021. "A Global Survey of Potential Acceptance of a COVID-19 Vaccine.” Nature Medicine 27 (2): 225-228.

May, Thomas, and Ross D. Silverman. 2005. "Free-Riding, Fairness, and the Rights of Minority Groups in Exemption from Mandatory Childhood Vaccination." Human Vaccines 1 (1): 12-15.

Moss, William J., and Peter Strebel. 2011. "Biological Feasibility of Measles Eradication." The Journal of Infectious Diseases 204 (Supplement_1): S47-S53.

National Conference of State Legislatures. 2021. "States with Religious and Philosophical Exemptions from School Immunization Requirements." National Conference of State Legislatures. Accessed February 11, 2021. https://www.ncsl.org/research/ health/school-immunization-exemption-state-laws.aspx.

Navin, Mark. 2016. Values and Vaccine Refusal: Hard Questions in Ethics, Epistemology, and Health Care. New York, NY: Routledge.

Navin, Mark C., Mark A. Largent, and Aaron M. McCright. 2020. "Efficient Burdens Decrease Nonmedical Exemption Rates: A Cross-County Comparison of Michigan's Vaccination Waiver Education Efforts." Preventive Medicine Reports 17: 101049.

Olson, Jr., Mancur. 1965. The Logic of Collective Action: Public Goods and the Theory of Groups. Cambridge, MA: Harvard University Press. 
Omer, Saad B., Jennifer L. Richards, Michelle Ward, and Robert A. Bednarczyk. 2012. "Vaccination Policies and Rates of Exemption from Immunization, 2005-2011." The New England Journal of Medicine 367 (12): 1170-1171.

Omer, Saad B., Kyle S. Enger, Lawrence H. Moulton, Neal A. Halsey, Shannon Stokley, and Daniel A. Salmon. 2008. "Geographic Clustering of Nonmedical Exemptions to School Immunization Requirements and Associations with Geographic Clustering of Pertussis." American Journal of Epidemiology 168 (12): 1389-1396.

Phadke, Varun K., Robert A. Bednarczyk, Daniel A. Salmon, and Saad B. Omer. 2016. "Association Between Vaccine Refusal and Vaccine-Preventable Diseases in the United States: A Review of Measles and Pertussis." JAMA 315 (11): 1149-1158.

Pierik, Roland. 2018. "Mandatory Vaccination: An Unqualified Defence.” Journal of Applied Philosophy 35 (2): 381-398.

Quadri-Sheriff, Maheen, Kristin S. Hendrix, Stephen M. Downs, Lynne A. Sturm, Gregory D. Zimet, and S. Maria E. Finnell. 2012. "The Role of Herd Immunity in Parents' Decision to Vaccinate Children: A Systematic Review." Pediatrics 130 (3): 522-530.

Salmon, Daniel A., and Andrew W. Siegel. 2001. "Religious and Philosophical Exemptions from Vaccination Requirements and Lessons Learned from Conscientious Objectors from Conscription." Public Health Reports 116 (4): 289-295.

Schröder-Bäck, Peter, Helmut Brand, Ixhel Escamilla, John K. Davies, Caroline Hall, Kieran Hickey, Eleni Jelastopulu, Reli Mechtler, and Jaroslav Volf. 2009. "Ethical Evaluation of Compulsory Measles Immunisation as a Benchmark for Good Health Management in the European Union." Central European Journal of Public Health 17 (4): 183-186.

Sears, Robert W. 2007. The Vaccine Book: Making the Right Decision for Your Child. New York, NY: Little, Brown and Co.

Siegal, Gil, Neomi Siegal, and Richard J. Bonnie. 2009. “An Account of Collective Actions in Public Health." American Journal of Public Health 99 (9): 1583-1587.

Smith, Michael. 2015. "Vaccine Safety: Medical Contraindications, Myths, and Risk Communication." Pediatrics in Review 36 (6): 227-238.

Sobo, Elisa J. 2016. "What Is Herd Immunity, and How Does It Relate to Pediatric Vaccination Uptake? US Parent Perspectives.” Social Science \& Medicine 165: 187-195.

Spencer, Jeanne P., Ruth H. Trondsen Pawlowski, and Stephanie Thomas. 2017. "Vaccine Adverse Events: Separating Myth from Reality." American Family Physician 95 (12): 786-794.

van den Hoven, Mariëtte. 2012. "Why One Should Do One’s Bit: Thinking About Free Riding in the Context of Public Health Ethics.” Public Health Ethics 5 (2): 154-160.

van Rooij, Benjamin, Anne Leonore de Bruijn, Chris Reinders Folmer, Emmeke Barbara Kooistra, Malouke Esra Kuiper, Megan Brownlee, Elke Olthuis, and Adam Fine. 2020. "Compliance with COVID-19 Mitigation Measures in the United States." Amsterdam Law School Research Paper No. 2020-21. Social Science Research Network, Rochester, NY.

Vaz, Olivia M., Mallory K. Ellingson, Paul Weiss, Samuel M. Jenness, Azucena Bardají, Robert A. Bednarczyk, and Saad B. Omer. 2020. "Mandatory Vaccination in Europe." Pediatrics 145 (2): e20190620. 
Ethan Bradley is an undergraduate student of philosophy and political science at Oakland University (Rochester, MI, USA).

Contact e-mail: <embradley@oakland.edu>

Mark Navin is professor and chair of philosophy at Oakland University (Rochester, MI, USA).

Contact e-mail: <navin@oakland.edu> 